\title{
Approximate-analytical solutions of cable equation using conformable fractional operator
}

\author{
Mehmet Yavuz and Burcu Yaşkıran \\ Department of Mathematics-Computer Sciences, Necmettin Erbakan University, Meram, Konya, Turkey
}

Received: 11 November 2017, Accepted: 11 December 2017

Published online: 12 December 2017.

\begin{abstract}
In the present work, we have introduced a new formulation for the approximate-analytical solution of the fractional onedimensional cable differential equation (FCE) by using the conformable fractional derivative. First of all, we have redefined Adomian decomposition method (CADM) and variational iteration method (CVIM) in the conformable sense. Then, we have solved by using the mentioned methods, which can analytically solve the fractional partial differential equations (FPDEs). In order to represent the efficiencies of these proposed methods, we have compared the numerical and exact solutions of the (FCE). Also, we have found out that the proposed models defined with the conformable derivative operator are very efficient and powerful techniques in finding approximate- analytical solutions for the cable equation of fractional order. In addition, the classical derivative and integral properties are recovered partially when the fractional term (alpha) is equal to one.
\end{abstract}

Keywords: Conformable derivative operator, approximate-analytical solution, Adomian decomposition method, variational iteration method, fractional cable equation.

\section{Introduction}

Fractional differential equations have been used to model problems in viscoelastic materials, fluid mechanics, biology, physics, finance, bioengineering and other areas of application [1,2,3,4,5,6]. There are many studies on Adomian decomposition method (ADM) which can evaluate the solutions of FPDEs. The ADM has been applied to obtain approximate solutions of linear or nonlinear fractional differential equations, fractional ordinary differential equations (FODEs), FPDEs, integral and integro-differential equations [7,8,9,10,11,12,13,14,15]. Meanwhile, there are many applications of solution methods based on variational iteration method (VIM) to ordinary-partial differential equations and other research areas [16,17,18,19,20]. In addition, Zhang [21] applied a finite difference method for the FPDEs. Meerschaert and Tadjeran [22] used finite difference approximations for two-sided space-fractional PDEs. Shawagfeh [23] explained analytical approximate solutions for nonlinear fractional differential equations. Recently, [24, 25, 26,27] recommended a new fractional derivative called conformable derivative operator (CDO) and by means of this operator, some solution methods have been improved. Many researchers [28,29,30,31,32,33] have applied the CDO to the problems in engineering, finance, biology, medicine, physics and applied mathematics. In this study, we have solved the fractional cable equation using the proposed methods described by using the conformable fractional derivative.

The fractional cable equation can be given in its general form as [34].

$$
\frac{\partial u(x, t)}{\partial t}={ }_{0} D_{t}^{1-\gamma_{1}}\left(K \frac{\partial^{2} u(x, t)}{\partial x^{2}}\right)+\mu_{00}^{2} D_{t}^{1-\gamma_{2}} u(x, t)+f(x, t)
$$


with the initial condition

$$
u(x, 0)=g(x), 0 \leq x \leq L,
$$

and the boundary conditions

$$
u(0, t)=\varphi(t), u(L, t)=\psi(t), 0 \leq t \leq T,
$$

where $0<\gamma_{1}, \gamma_{2}<1, K>0$ and $\mu_{0}^{2}$ are constants, and ${ }_{0} D_{t}^{1-\gamma_{1}} u(x, t)$ is the conformable fractional derivative operator of order $1-\gamma_{1}$. In the literature, there are some processes of approximate solutions of the FCE. Implicit numerical methods (INM) [34], the implicit compact difference scheme (ICDS) [35], and explicit numerical methods (ENM) [36] have been applied to the FCE.

In this study, we consider the following non-homogeneous fractional cable equation for the special case:

$$
\frac{\partial u(x, t)}{\partial t}={ }_{0} D_{t}^{1-\alpha} \frac{\partial^{2} u(x, t)}{\partial x^{2}}-{ }_{0} D_{t}^{1-\alpha} u(x, t)+f(x, t), 0<\alpha \leq 1,
$$

with the initial condition

$$
u(x, 0)=0,0 \leq x \leq 1,
$$

and the boundary conditions

$$
u(0, t)=0, u(1, t)=0,0 \leq t \leq T,
$$

where $f(x, t)=2 \sin \pi x\left(t+\left(\pi^{2}+1\right) \frac{t^{1+\alpha}}{\Gamma(\alpha+2)}\right)$. The exact solution of Eq. (1)-(3) is given by $u(x, t)=t^{2} \sin \pi x$ [34].

The main purpose of this study is to redefine ADM and VIM for the solution of the FCE by using the conformable derivative. We have solved FCE of fractional order using the suggested methods and we have compared the numerical and approximate-analytical solutions in term of figures and tables. Therefore, we have fulfilled the purpose. When looking at the results, it is obvious that these methods are very effective and accurate for solving fractional cable differential equation (FCDE).

\section{Conformable derivative operator}

Definition 1. Given a function $f:[0, \infty) \rightarrow \mathrm{R}$. Then the conformable derivative of $f$ order $\alpha \in(0,1]$ is defined by [24]:

$$
D_{* t}^{\alpha}(f)(t)=\lim _{\varepsilon \rightarrow 0} \frac{f\left(t+\varepsilon t^{1-\alpha}\right)-f(t)}{\varepsilon},
$$

for all $t>0$.

Theorem 1. [24] Let $\alpha \in(0,1]$ and $f, g$ be $\alpha$-differentiable at a point $t>0$. Then;

(1) $D_{* t}^{\alpha}(a f+b g)=a D_{* t}^{\alpha}(f)+b D_{* t}^{\alpha}(g)$ for all $a, b \in \mathrm{R}$,

(2) $D_{* t}^{\alpha}\left(t^{k}\right)=k t^{k-\alpha}$ for all $k \in \mathrm{R}$,

(3) $D_{* t}^{\alpha}(f(t))=0$ for all constant functions $f(t)=k$,

(4) $D_{* t}^{\alpha}(f g)=f D_{* t}^{\alpha}(g)+g D_{* t}^{\alpha}(f)$,

(5) $D_{* t}^{\alpha}(f / g)=\frac{g D_{* t}^{\alpha}(f)-f D_{* t}^{\alpha}(g)}{g^{2}}$,

(6) If $f(t)$ is differentiable, then $D_{* t}^{\alpha}(f(t))=t^{1-\alpha} \frac{d}{d t} f(t)$.

Definition 2. [24,25] Let $f$ be an n-times differentiable at $t$. Then the conformable derivative of $f$ order $\alpha$ is defined as:

$$
D_{* t}^{\alpha}(f(t))=\lim _{\varepsilon \rightarrow 0} \frac{f^{(\lceil\alpha\rceil-1)}\left(t+\varepsilon t^{(\lceil\alpha\rceil-\alpha)}\right)-f^{(\lceil\alpha\rceil-1)}(t)}{\varepsilon},
$$


for all $t>0, \alpha \in(n, n+1$ Here $\lceil\alpha\rceil$ is the smallest integer greater than or equal to $\alpha$.

Lemma 1. [24,25] Let $f$ be an $n$-times differentiable at $t$. Then

$$
D_{* t}^{\alpha}(f(t))=t^{\lceil\alpha\rceil-\alpha} f^{\lceil\alpha\rceil}(t)
$$

for all $t>0, \alpha \in(n, n+1]$.

\section{Adomian Decomposition Method in Conformable Sense}

Consider the following nonlinear fractional partial differential equation:

$$
L_{\alpha}(u(x, t))+R(u(x, t))+N(u(x, t))=v(x, t),
$$

where $L_{\alpha}=D_{* t}^{\alpha}$ is a linear operator with the conformable derivative of order $\alpha(n<\alpha \leq n+1), R$ is the another part of the linear operator, $N$ is a non-linear operator and $v(x, t)$ is a non-homogeneous term. In Eq. (7), if we apply the linear operator to Lemma 1 , we obtain the following equation :

$$
t^{\lceil\alpha\rceil-\alpha} \frac{\partial^{\lceil\alpha\rceil} u(x, t)}{\partial t^{\lceil\alpha\rceil}}+R(u(x, t))+N(u(x, t))=v(x, t) .
$$

Applying the inverse of linear operator $L_{\alpha}^{-1}=\int_{0}^{t} \int_{0}^{\gamma_{1}} \cdots \int_{n}^{\gamma_{n-1}} \frac{1}{\gamma_{n}^{\alpha \mid-\alpha}}(.) d \gamma_{n} d \gamma_{n-1} \cdots d \gamma_{1}$, to both sides of Eq. (7), we obtain

$$
L_{\alpha}^{-1} L_{\alpha}(u(x, t))+L_{\alpha}^{-1} R(u(x, t))+L_{\alpha}^{-1} N(u(x, t))=L_{\alpha}^{-1} v(x, t) .
$$

According to the conformable ADM, we can demonstrate the solution $u(x, t)$ with the infinite series of components

$$
u(x, t)=\sum_{n=0}^{\infty} u_{n}(x, t)
$$

The nonlinear term dissociated in Eq. (7) is given by [37]:

$$
N(u)=\sum_{n=0}^{\infty} A_{n},\left(u_{0}, u_{1}, \cdots, u_{n}\right)
$$

where $A_{n}$ is the so-called Adomian polynomials. These polynomials can be derived for all nonlinear term with respect to the algorithms developed by Adomian.

Substituting (9) and (10) into (8), we obtain

$$
\sum_{n=0}^{\infty} u_{n}=u(x, 0)+L_{\alpha}^{-1} v-L_{\alpha}^{-1} R\left(\sum_{n=0}^{\infty} u_{n}\right)-L_{\alpha}^{-1}\left(\sum_{n=0}^{\infty} A_{n}\right)
$$

By using Eq. (11), the iteration terms are obtained by the following way:

$$
\begin{aligned}
u_{0}= & u(x, 0)+L_{\alpha}^{-1} v \\
u_{1}=- & L_{\alpha}^{-1} R u_{0}-L_{\alpha}^{-1} A_{0}, \\
& \vdots \\
u_{n+1}= & -L_{\alpha}^{-1} R u_{n}-L_{\alpha}^{-1} A_{n}, n \geq 0 .
\end{aligned}
$$


Then, the approximate-analytical solution of Eq. (7) is obtained by

$$
\tilde{u}_{k}(x, t)=\sum_{n=0}^{k} u_{n}(x, t)
$$

Finally, we obtain the exact solution of Eq. (7) as

$$
u(x, t)=\lim _{k \rightarrow \infty} \tilde{u}_{k}(x, t)
$$

\section{Variational iteration method in conformable sense}

In this section, it is given some basic solution steps and properties of variational iteration method in conformable sense (CVIM) definition. We introduce a solution algorithm in an effective way for the nonlinear FPDEs of fractional order. Firstly, we consider the following nonlinear fractional equation:

$$
D_{* t}^{\alpha} u(x, t)+L u(x, t)+N u(x, t)=v(x, t), \quad t>0,
$$

where $L$ is a linear operator, $N$ is a nonlinear operator, $v$ is a known analytical function and $D_{* t}^{\alpha}$ is conformable fractional derivative of order $\alpha$ with $0<\alpha \leq 1$. In Eq. (13), if we apply the linear operator to Lemma 1, we get

$$
t^{\lceil\alpha\rceil-\alpha} \frac{\partial^{\lceil\alpha\rceil} u(x, t)}{\partial t^{\lceil\alpha\rceil}}+L\left(u, u_{x}, u_{x x}\right)+N\left(u, u_{x}, u_{x x}\right)=v(x, t) .
$$

According to the variation technique, the correction functional can be constructed as [38]

$$
u_{n+1}(x, t)=u_{n}(x, t)+\int_{0}^{t} \lambda(\zeta)\left[\zeta^{1-\alpha} \frac{\partial u_{n}(x, \zeta)}{\partial \zeta}+L\left(u_{n}(x, \zeta)\right)+N\left(\tilde{u}_{n}(x, \zeta)\right)-v(x, \zeta)\right] d \zeta
$$

where $\lambda$ is the Lagrange multiplier, that in this method may be a constant or a function, Taking the variation of both sides of Eq. (14) with respect to the independent variable $u$, we find

$$
\delta u_{n+1}(x, t)=\delta u_{n}(x, t)+\delta\left(\int_{0}^{t} \lambda(\zeta)\left[\zeta^{1-\alpha} \frac{\partial u_{n}(x, \zeta)}{\partial \zeta}+L\left(u_{n}(x, \zeta)\right)+N\left(\tilde{u}_{n}(x, \zeta)\right)-v(x, \zeta)\right] d \zeta\right)
$$

and $\tilde{u}_{n}$ is a restricted value where $\delta \tilde{u}_{n}=0$. For $\alpha=1$ we obtain for Eq. (15) the following stationary conditions

$$
1+\left.\lambda(t)\right|_{\zeta=t}=0, \quad \lambda^{\prime}(\zeta)=0
$$

Therefore the general Lagrange multipliers, can be specified as $\lambda(\zeta)=-1$. So, we get the iteration formula in the following form

$$
u_{n+1}(x, t)=u_{n}(x, t)-\int_{0}^{t}\left[\zeta^{1-\alpha} \frac{\partial u_{n}(x, \zeta)}{\partial \zeta}+L\left(u_{n}(x, \zeta)\right)+N\left(\tilde{u}_{n}(x, \zeta)\right)-v(x, \zeta)\right] d \zeta
$$

By using the determined Lagrange multiplier and any selected function $u_{0}, u_{n+1}$, which is the successive approximations of $u(x)$ for $n \geq 0$, will be obtained readily. Hence, we get the solution as

$$
u(x)=\lim _{n \rightarrow \infty} u_{n}(x) .
$$




\section{Solution of the fractional cable equation}

\subsection{Solution by CADM}

Now let us solve the problem in Eq. (4) with the initial condition (5) and the boundary conditions (6) by using Adomian decomposition method considering the conformable derivative operator.

Let $L_{\alpha}=D_{* t}^{\alpha}=\frac{\partial^{\alpha}}{\partial t^{\alpha}}=t^{1-\alpha} \frac{\partial}{\partial t}$ be a linear operator, then if we apply the operator to Eq. (4) we have

$$
D_{* t}^{\alpha} u(x, t)=\frac{\partial^{2} u(x, t)}{\partial x^{2}}-u(x, t)+D_{* t}^{\alpha} D^{-1} f(x, t) .
$$

By using the Lemma 1, we can write the Eq. (17) as

$$
t^{1-\alpha} \frac{\partial u(x, t)}{\partial t}=\frac{\partial^{2} u(x, t)}{\partial x^{2}}-u(x, t)+t^{1-\alpha} \frac{\partial}{\partial t} D^{-1} f(x, t) .
$$

Now, we apply the inverse of operator $L_{\alpha}$ which is $L_{\alpha}^{-1}=\int_{0}^{t} \frac{1}{\zeta^{1-\alpha}}(). d \zeta$ to both sides of Eq. (18), we get

$$
u(x, t)=u(x, 0)+L_{\alpha}^{-1}\left(t^{1-\alpha} f(x, t)\right)+L_{\alpha}^{-1}\left(\frac{\partial^{2} u(x, t)}{\partial x^{2}}-u(x, t)\right) .
$$

According to the iteration terms Eq. (12) and the initial condition Eq. (5), we can write the decomposition series terms. Therefore, the other components of the series are given by:

$$
\begin{aligned}
& u_{0}=u(x, 0)+L_{\alpha}^{-1}\left(t^{1-\alpha} f(x, t)\right)=2 \sin \pi x\left(\frac{t^{2}}{2}+\frac{\left(\pi^{2}+1\right) t^{\alpha+2}}{\Gamma(3+\alpha)}\right), \\
& u_{1}=L_{\alpha}^{-1}\left(\frac{\partial^{2} u_{0}}{\partial x^{2}}-u_{0}\right)=-2 \sin \pi x\left(\frac{\left(\pi^{2}+1\right) t^{\alpha+2}}{2(\alpha+2)}+\frac{\left(\pi^{2}+1\right)^{2} t^{2 \alpha+2}}{(2 \alpha+2) \Gamma(3+\alpha)}\right), \\
& u_{2}=L_{\alpha}^{-1}\left(\frac{\partial^{2} u_{1}}{\partial x^{2}}-u_{1}\right)=2 \sin \pi x\left(\frac{\left(\pi^{2}+1\right)^{2} t^{2 \alpha+2}}{2(\alpha+2)(2 \alpha+2)}+\frac{\left(\pi^{2}+1\right)^{3} t^{3 \alpha+2}}{(2 \alpha+2)(3 \alpha+2) \Gamma(3+\alpha)}\right), \\
& \vdots \\
& u_{n}=L_{\alpha}^{-1}\left(\frac{\partial^{2} u_{n-1}}{\partial x^{2}}-u_{n-1}\right)=2(-1)^{n} \sin \pi x\left(\frac{\left(\pi^{2}+1\right)^{n} t^{n \alpha+2}}{2(\alpha+2)(2 \alpha+2) \cdots(n \alpha+2)}+\frac{\left(\pi^{2}+1\right)^{n+1} t^{(n+1) \alpha+2}}{(2 \alpha+2)(3 \alpha+2) \cdots((n+1) \alpha+2) \Gamma(3+\alpha)}\right),
\end{aligned}
$$

and such like, we can calculate the rest of components of the Adomian series. Then the approximate solution of Eq. (4) is given by

$$
\tilde{u}_{k}(x, t)=\sum_{n=0}^{k} u_{n}(x, t) .
$$

Using the last equation we obtain the approximate analytical solution of the proposed problem

$$
u(x, t) \cong 2 \sin \pi x\left[\frac{t^{2}}{2}\left(1-\frac{\left(\pi^{2}+1\right) t^{\alpha}}{\alpha+2-\left(\pi^{2}+1\right) t^{\alpha}}\right)+\frac{t^{2}}{\Gamma(2+\alpha)} \frac{\left(\pi^{2}+1\right) t^{\alpha}}{\alpha+2-\left(\pi^{2}+1\right) t^{\alpha}}\right] .
$$

Then the exact solution of the Eq. (4) subject to the initial condition (5) and the boundary conditions (6) for special case of $\alpha=1$, is obtained with CADM as

$$
u(x, t) \cong t^{2} \sin \pi x .
$$

In the following Figure 1, CADM solution results of the cable equation for various values of the distance term $x$ and the time variable $t$ for special case of $\alpha=0.30$ (in left) and $\alpha=0.70$ (in right). 

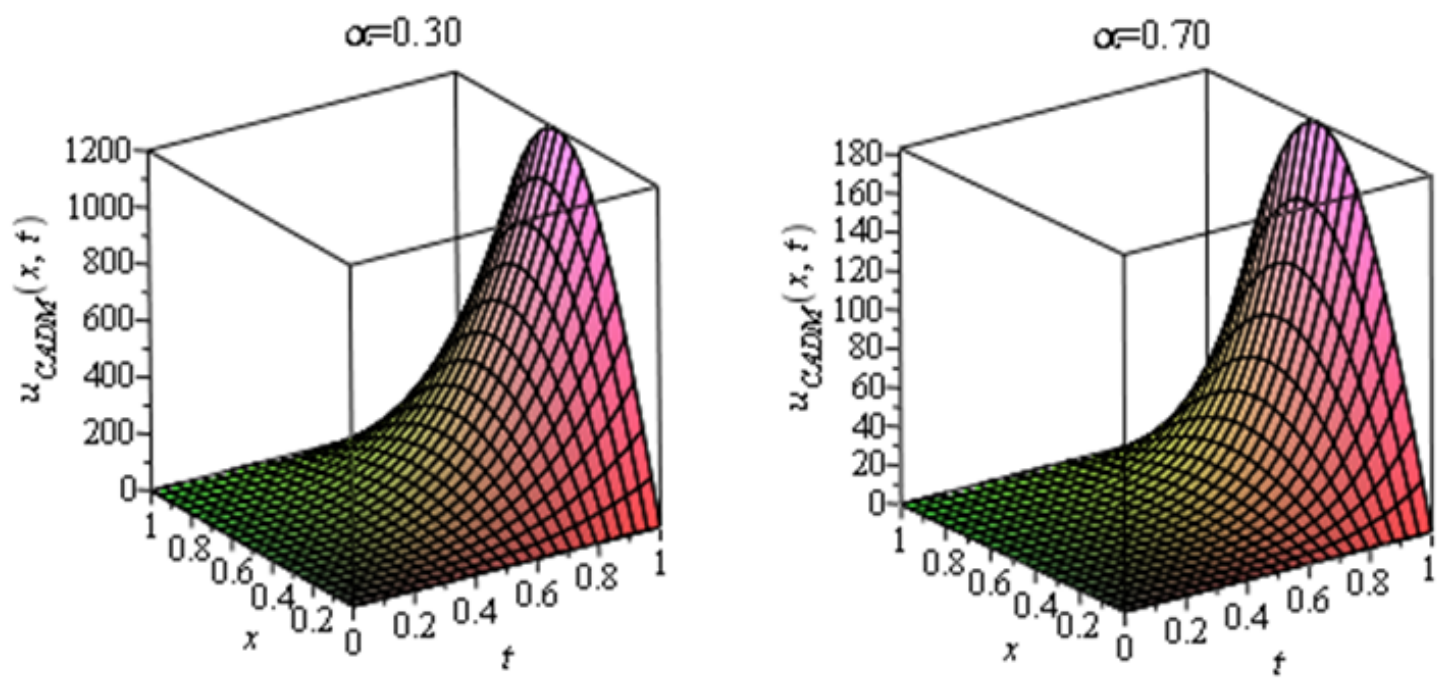

Fig. 1: CADM solutions of the cable equation with $\alpha=0.30$ and $\alpha=0.70$.

\subsection{Solution by CVIM}

In order to solve the Eq. (13) by way of conformable VIM, we have used the iteration formula (14) and the iteration formula of the solution is given by:

$$
u_{n+1}(x, t)=u_{n}(x, t)-\int_{0}^{t}\left[\zeta^{1-\alpha} \frac{\partial u_{n}(x, \zeta)}{\partial \zeta}-\frac{\partial^{2} u_{n}(x, \zeta)}{\partial x^{2}}+u_{n}(x, \zeta)-\zeta^{1-\alpha} \frac{\partial}{\partial \zeta} D_{\zeta}^{-1} f(x, \zeta)\right] d \zeta
$$

In addition, by using the Eq. (20) we can obtain the following set of linear PDEs:

$$
\begin{aligned}
u_{0} & =u(x, 0)=0, \\
u_{1} & =u_{0}-\int_{0}^{t}\left[\zeta^{1-\alpha} \frac{\partial u_{0}(x, \zeta)}{\partial \zeta}-\frac{\partial^{2} u_{0}(x, \zeta)}{\partial x^{2}}+u_{0}(x, \zeta)-\zeta^{1-\alpha} \frac{\partial}{\partial \zeta} D^{-1} f(x, \zeta)\right] d \zeta \\
& =2 \sin \pi x\left(\frac{t^{3-\alpha}}{3-\alpha}+\left(\pi^{2}+1\right) \frac{t^{3}}{3 \Gamma(2+\alpha)}\right), \\
u_{2} & =u_{1}-\int_{0}^{t}\left[\zeta^{1-\alpha} \frac{\partial u_{1}(x, \zeta)}{\partial \zeta}-\frac{\partial^{2} u_{1}(x, \zeta)}{\partial x^{2}}+u_{1}(x, \zeta)-\zeta^{1-\alpha} \frac{\partial}{\partial \zeta} D^{-1} f(x, \zeta)\right] d \zeta \\
& =4 \sin \pi x\left(\frac{t^{3-\alpha}}{3-\alpha}+\frac{\left(\pi^{2}+1\right) t^{3}}{3 \Gamma(2+\alpha)}\right)-2 \sin \pi x\left(\frac{t^{4-2 \alpha}}{4-2 \alpha}+\frac{\left(\pi^{2}+1\right) t^{4-\alpha}}{(4-\alpha) \Gamma(2+\alpha)}\right) \\
& -2 \sin \pi x\left(\frac{\left(\pi^{2}+1\right) t^{4-\alpha}}{(4-\alpha)(3-\alpha)}+\frac{\left(\pi^{2}+1\right)}{4 \cdot 3 \cdot \Gamma(2+\alpha)}\right), \\
u_{3} & =u_{2}-\int_{0}^{t}\left[\zeta^{1-\alpha} \frac{\partial u_{2}(x, \zeta)}{\partial \zeta}-\frac{\partial^{2} u_{2}(x, \zeta)}{\partial x^{2}}+u_{2}(x, \zeta)-\zeta^{1-\alpha} \frac{\partial}{\partial \zeta} D^{-1} f(x, \zeta)\right] d \zeta \\
& =6 \sin \pi x\left(\frac{t^{3-\alpha}}{3-\alpha}+\frac{\left(\pi^{2}+1\right) t^{3}}{3 \Gamma(2+\alpha)}\right)-6 \sin \pi x\left(\frac{t^{4-2 \alpha}}{4-2 \alpha}+\frac{\left(\pi^{2}+1\right) t^{4-\alpha}}{(4-\alpha) \Gamma(2+\alpha)}\right)
\end{aligned}
$$




$$
\begin{aligned}
& -6 \sin \pi x\left(\frac{\left(\pi^{2}+1\right) t^{4-\alpha}}{(4-\alpha)(3-\alpha)}+\frac{\left(\pi^{2}+1\right)^{2} t^{4}}{4 \cdot 3 \cdot \Gamma(2+\alpha)}\right)+2 \sin \pi x\left(\frac{t^{5-3 \alpha}}{5-3 \alpha}+\frac{\left(\pi^{2}+1\right) t^{5-2 \alpha}}{(5-2 \alpha) \Gamma(2+\alpha)}\right) \\
& +2 \sin \pi x\left(\frac{\left(\pi^{2}+1\right) t^{5-2 \alpha}}{(5-2 \alpha)(3-\alpha)}+\frac{\left(\pi^{2}+1\right)^{2} t^{5-\alpha}}{3(5-\alpha) \Gamma(2+\alpha)}\right)+2 \sin \pi x\left(\frac{\left(\pi^{2}+1\right) t^{5-2 \alpha}}{(5-2 \alpha)(4-2 \alpha)}+\frac{\left(\pi^{2}+1\right)^{2} t^{5-\alpha}}{(5-\alpha)(4-\alpha) \Gamma(2+\alpha)}\right) \\
& +2 \sin \pi x\left(\frac{\left(\pi^{2}+1\right)^{2} t^{5-\alpha}}{(5-\alpha)(4-\alpha)(3-\alpha)}+\frac{\left(\pi^{2}+1\right)^{3} t^{5}}{5 \cdot 4 \cdot 3 \cdot \Gamma(2+\alpha)}\right)
\end{aligned}
$$

and so on. When we consider $\alpha=1$, the solution obtained by variational iteration method in the conformable mean is given by

$$
u_{n}(x, t)=t^{2} \sin \pi x\left(\frac{6}{2}+\left(\pi^{2}+1\right) \frac{6 t}{3 \Gamma(3)}-\frac{6}{2}-\left(\pi^{2}+1\right) \frac{6 t}{3 \Gamma(3)}-\left(\pi^{2}+1\right) \frac{6 t}{3 \cdot 2}-\left(\pi^{2}+1\right) \frac{6 t^{2}}{4 \cdot 3 \cdot \Gamma(3)}+\cdots\right)
$$

By using the equations in (21), the exact solution of the CVIM solution for special case of $\alpha=1$ is given as follows:

$$
u(x, t)=t^{2} \sin \pi x
$$

The following Figure 2 shows the CVIM solutions results of the cable equation for $(x, t)=[0,1] \times[0,1]$ considering the fractional operator $\alpha=0.30$ (in left) and $\alpha=0.70$ (in right).
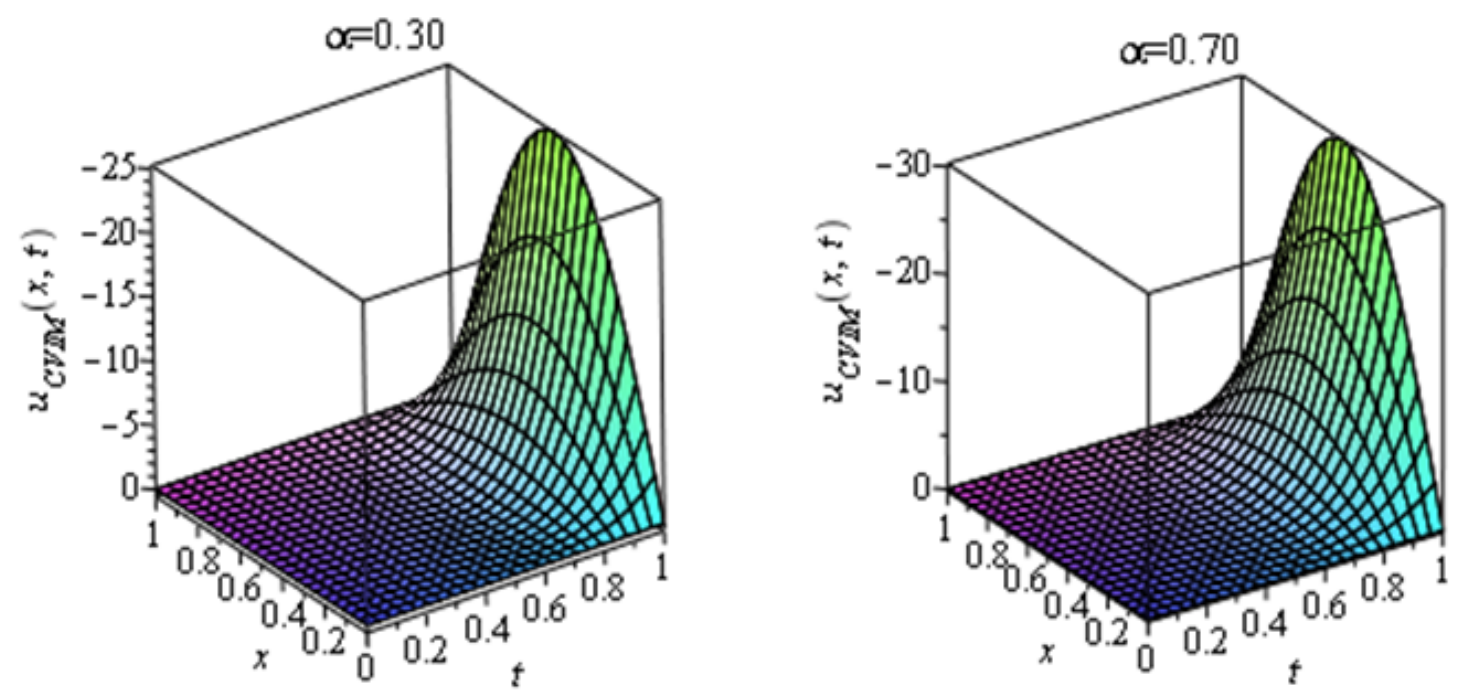

Fig. 2: CVIM solutions of the cable equation with $\alpha=0.30$ and $\alpha=0.70$.

According to Table 1 we can observe that the absolute error values in terms of the solution of the Eq. (4) are very small for various values $\alpha, x$ and $t$. It is very important to characterize the behavior of the error and to make the error stability of the method used for numerical solutions. Therefore, we have investigated the error analysis of the recommended methods and we have shown the absolute error values in Figure 3. Moreover, we have presented in Figure 4 the comparison of the numerical and exact solutions. Figure 3 and Figure 4 have declared that the solutions obtained using CVIM is closer to the exact solutions with respect to CADM ones. 
Table 1: Absolute errors $\left|\tilde{u}_{k}(x, t)-u(x, t)\right|$ obtained with CADM and CVIM for the cable equation.

\begin{tabular}{|c|c|c|c|c|c|c|c|}
\hline \multirow{2}{*}{$x$} & \multirow{2}{*}{$t$} & \multicolumn{2}{|c|}{$\alpha=0.35$} & \multicolumn{2}{c|}{$\alpha=0.75$} & \multicolumn{2}{c|}{$\alpha=0.95$} \\
\cline { 3 - 8 } & & CADM & CVIM & CADM & CVIM & CADM & CVIM \\
\hline \multirow{3}{*}{$10^{-3}$} & 0.10 & 0.00046588 & 0.00000281 & 0.00000076 & 0.00000042 & 0.00000004 & 0.00000000 \\
& 0.50 & 13.5023030 & 13.7112263 & 0.00847201 & 0.00038284 & 0.00206843 & 0.00143082 \\
& 0.70 & 322.724387 & 323.473669 & 0.05919425 & 0.00774314 & 0.02011917 & 0.01250838 \\
\hline \multirow{3}{*}{$10^{-5}$} & 0.10 & 0.00000466 & 0.00000003 & 0.00000001 & 0.00000000 & 0.00000000 & 0.00000000 \\
& 0.50 & 0.13502325 & 0.13711249 & 0.00008472 & 0.00000383 & 0.00002068 & 0.00001431 \\
& 0.70 & 3.22724918 & 3.23474201 & 0.00059194 & 0.00007743 & 0.00020119 & 0.00012508 \\
\hline \multirow{4}{*}{$10^{-6}$} & 0.10 & 0.00000047 & 0.00000000 & 0.00000000 & 0.00000000 & 0.00000000 & 0.00000000 \\
& 0.50 & 0.01350233 & 0.01371125 & 0.00000847 & 0.00000038 & 0.00000207 & 0.00000143 \\
& 0.70 & 0.32272492 & 0.32347420 & 0.00005919 & 0.00000774 & 0.00002012 & 0.00001251 \\
\hline \multirow{4}{*}{$10^{-8}$} & 0.10 & 0.00000000 & 0.00000000 & 0.00000000 & 0.00000000 & 0.00000000 & 0.00000000 \\
& 0.50 & 0.00013502 & 0.00013711 & 0.00000008 & 0.00000001 & 0.00000002 & 0.00000001 \\
& 0.70 & 0.00322725 & 0.00323474 & 0.00000059 & 0.00000008 & 0.00000020 & 0.00000013 \\
\hline
\end{tabular}

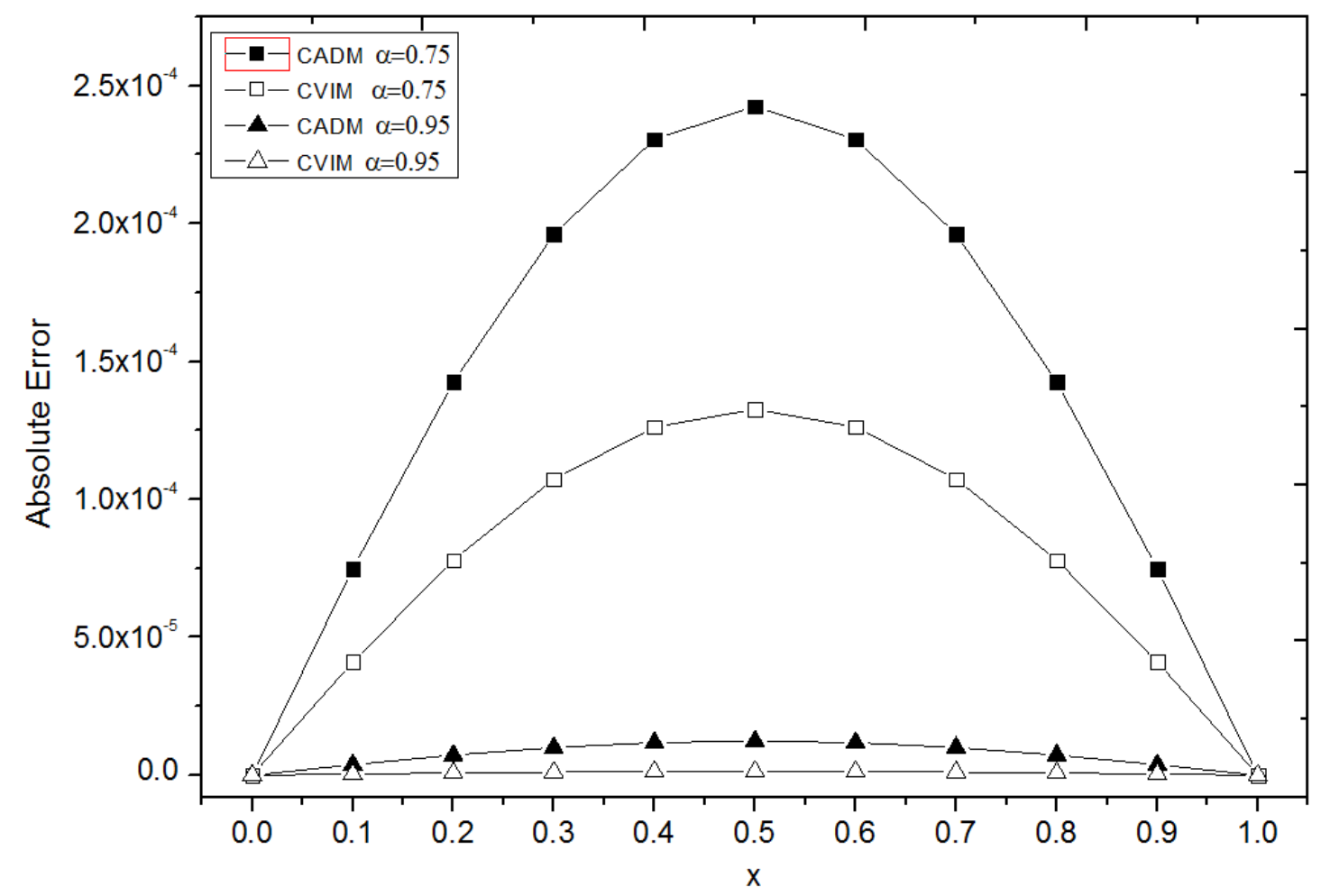

Fig. 3: Absolute error graph of CADM and CVIM at $t=0.1$ for various values of $\alpha$. 


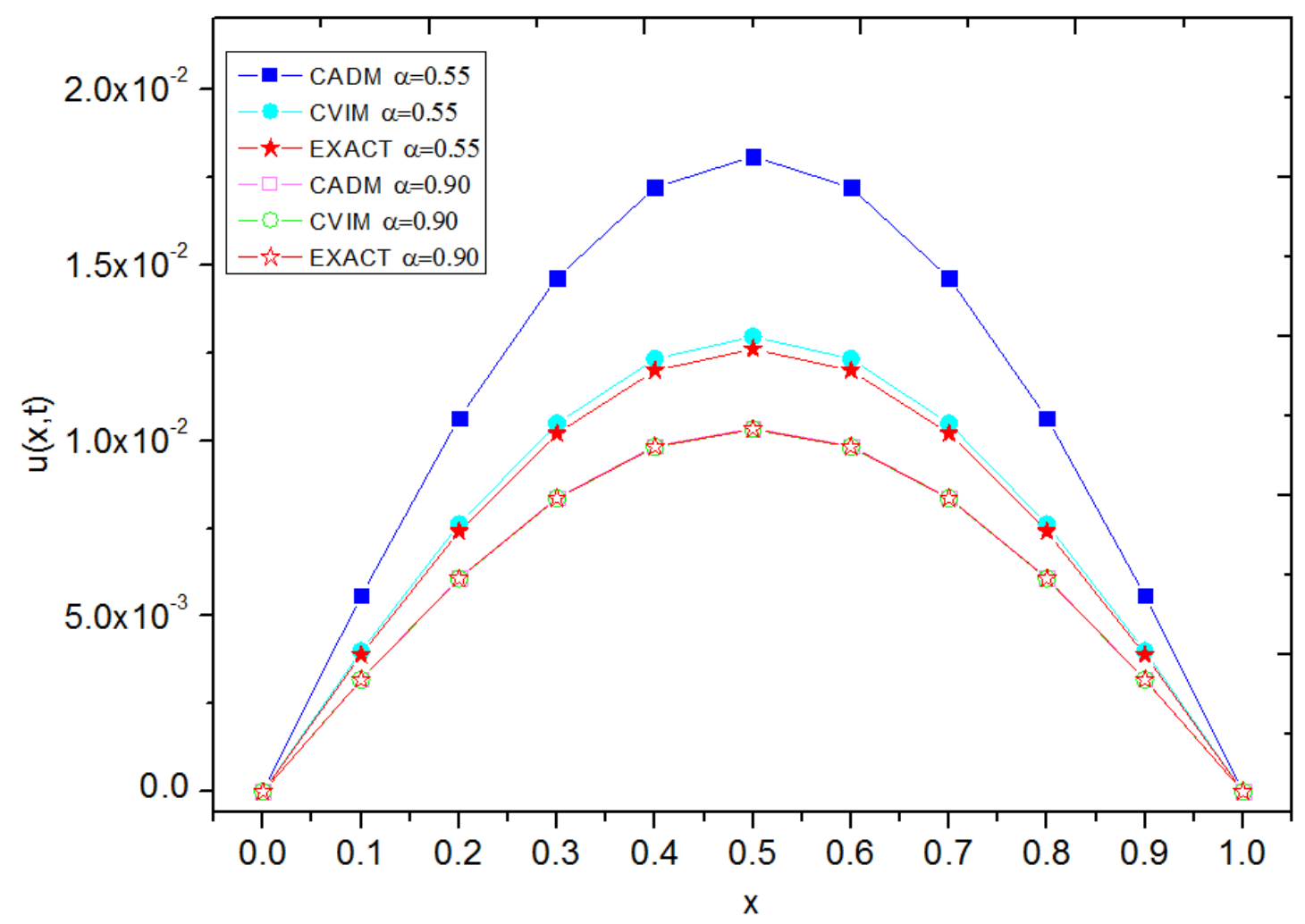

Fig. 4: Comparison the numerical solutions and the exact solutions at $t=0.1$ for various values of $\alpha$.

\section{Conclusion}

In the present paper, approximate-analytical solutions with two numerical methods for linear non-homogeneous time-fractional cable differential equation have been obtained. These methods are based on conformable derivative (CD) which is extremely popular in the last years. We have verified the efficiencies and accuracies of the suggested methods by applying them to the biological cable equation with the initial condition and the boundary conditions. The successful applications of the recommended models have indicated that these models are in complete settlement with the corresponding exact solutions. According to the solution graphs and tables, we can conclude that the solutions obtained using CVIM is closer to the exact solutions with respect to CADM ones. Meanwhile, in view of their usability, our methods are applicable to many initial-boundary value problems and linear-nonlinear partial differential equations of fractional order.

\section{Competing interests}

The authors declare that they have no competing interests.

\section{Authors' contributions}

All authors have contributed to all parts of the article. All authors read and approved the final manuscript. 


\section{References}

[1] Alkahtania, B. S. T., et al., New Numerical Analysis of Riemann-Liouville Time-Fractional Schrodinger with Power, Exponential Decay, and Mittag-Leffler Laws, Journal of Nonlinear Sciences and Applications, 10(8), 4231-4243, (2017).

[2] Evirgen, F. and N. Özdemir, A Fractional Order Dynamical Trajectory Approach for Optimization Problem with Hpm, in: Fractional Dynamics and Control, (Ed. D. Baleanu, Machado, J.A.T., Luo, A.C.J.), Springer, 145-155, (2012).

[3] Momani, S. and Z. Odibat, Analytical Approach to Linear Fractional Partial Differential Equations Arising in Fluid Mechanics, Physics Letters A, 355(4), 271-279, (2006).

[4] Özdemir, N. and M. Yavuz, Numerical Solution of Fractional Black-Scholes Equation by Using the Multivariate Padé Approximation, Acta Physica Polonica A, 132(3), 1050-1053, (2017).

[5] Turut, V. and N. Güzel, Multivariate Pade Approximation for Solving Nonlinear Partial Differential Equations of Fractional Order, Abstract and Applied Analysis, 2013, (2013).

[6] Yavuz, M., et al., Generalized Differential Transform Method for Fractional Partial Differential Equation from Finance, Proceedings, International Conference on Fractional Differentiation and its Applications, Novi Sad, Serbia, 778-785, (2016).

[7] Duan, J.-S., et al., A Review of the Adomian Decomposition Method and Its Applications to Fractional Differential Equations, Communications in Fractional Calculus, 3(2), 73-99, (2012).

[8] Wazwaz, A.-M., A New Algorithm for Calculating Adomian Polynomials for Nonlinear Operators, Applied Mathematics and Computation, 111(1), 33-51, (2000).

[9] Adomian, G., A Review of the Decomposition Method and Some Recent Results for Nonlinear Equations, Mathematical and Computer Modelling, 13(7), 17-43, (1990).

[10] Bildik, N. and H. Bayramoglu, The Solution of Two Dimensional Nonlinear Differential Equation by the Adomian Decomposition Method, Applied mathematics and computation, 163(2), 519-524, (2005).

[11] Bildik, N., et al., Solution of Different Type of the Partial Differential Equation by Differential Transform Method and Adomian's Decomposition Method, Applied Mathematics and Computation, 172(1), 551-567, (2006).

[12] Daftardar-Gejji, V. and H. Jafari, Adomian Decomposition: A Tool for Solving a System of Fractional Differential Equations, Journal of Mathematical Analysis and Applications, 301(2), 508-518, (2005).

[13] El-Sayed, A. and M. Gaber, The Adomian Decomposition Method for Solving Partial Differential Equations of Fractal Order in Finite Domains, Physics Letters A, 359(3), 175-182, (2006).

[14] El-Wakil, S., et al., Adomian Decomposition Method for Solving the Diffusion-Convection-Reaction Equations, Applied Mathematics and Computation, 177(2), 729-736, (2006).

[15] Evirgen, F. and N. Özdemir, Multistage Adomian Decomposition Method for Solving Nlp Problems over a Nonlinear Fractional Dynamical System, Journal of Computational and Nonlinear Dynamics, 6(2), 021003, (2011).

[16] Evirgen, F., Analyze the Optimal Solutions of Optimization Problems by Means of Fractional Gradient Based System Using Vim, An International Journal of Optimization and Control, 6(2), 75, (2016).

[17] He, J.-H., Variational Iteration Method: Some Recent Results and New Interpretations, Journal of Computational and Applied Mathematics, 207(1), 3-17, (2007).

[18] Turut, V. and N. Güzel, On Solving Partial Differential Equations of Fractional Order by Using the Variational Iteration Method and Multivariate Padé Approximations, European Journal of Pure and Applied Mathematics, 6(2), 147-171, (2013).

[19] İbiş, B. and M. Bayram, Approximate Solution of Time-Fractional Advection-Dispersion Equation Via Fractional Variational Iteration Method, The Scientific World Journal, 2014, (2014).

[20] İbiş, B. and M. Bayram, Analytical Approximate Solution of Time-Fractional Fornberg-Whitham Equation by the Fractional Variational Iteration Method, Alexandria Engineering Journal, 53(4), 911-915, (2014).

[21] Zhang, Y., A Finite Difference Method for Fractional Partial Differential Equation, Applied Mathematics and Computation, 215(2), 524-529, (2009).

[22] Meerschaert, M. M. and C. Tadjeran, Finite Difference Approximations for Two-Sided Space-Fractional Partial Differential Equations, Applied numerical mathematics, 56(1), 80-90, (2006).

[23] Shawagfeh, N. T., Analytical Approximate Solutions for Nonlinear Fractional Differential Equations, Applied Mathematics and Computation, 131(2), 517-529, (2002).

[24] Khalil, R., et al., A New Definition of Fractional Derivative, Journal of Computational and Applied Mathematics, 264, 65-70, (2014). 
[25] Anderson, D. and D. Ulness, Newly Defined Conformable Derivatives, Adv. Dyn. Syst. Appl, 10(2), 109-137, (2015).

[26] Atangana, A., et al., New Properties of Conformable Derivative, Open Mathematics, 13(1), (2015).

[27] Abdeljawad, T., On Conformable Fractional Calculus, Journal of computational and Applied Mathematics, 279, 57-66, (2015).

[28] Avc1, D., et al., Conformable Fractional Wave-Like Equation on a Radial Symmetric Plate, in: Theory and Applications of NonInteger Order Systems, (Ed. A. Babiarz, Czornik, A., Klamka, J., Niezabitowski, M.), Springer, 137-146, (2017).

[29] Avc1, D., et al., Conformable Heat Problem in a Cylinder, Proceedings, International Conference on Fractional Differentiation and its Applications, 572-581, (2016).

[30] Avci, D., et al., Conformable Heat Equation on a Radial Symmetric Plate, Thermal Science, 21(2), 819-826, (2017).

[31] Eroğlu, B. B. İ., et al., Optimal Control Problem for a Conformable Fractional Heat Conduction Equation, Acta Physica Polonica A, 132(3), 658-662, (2017).

[32] Evirgen, F., Conformable Fractional Gradient Based Dynamic System for Constrained Optimization Problem, Acta Physica Polonica A, 132(3), 1066-1069, (2017).

[33] Yavuz, M., Novel Solution Methods for Initial Boundary Value Problems of Fractional Order with Conformable Differentiation, An International Journal of Optimization and Control: Theories \& Applications (IJOCTA), 8(1), 1-7, (2018).

[34] Liu, F., et al., Stability and Convergence of Two New Implicit Numerical Methods for Fractional Cable Equation, Proceedings, Proceeding of the ASME 2009 International Design Engineering Technical Conferences \& Computers and Information in Engineering Conference, IDETC/CIE, San Diego, California, USA, (2009).

[35] Hu, X. and L. Zhang, Implicit Compact Difference Schemes for the Fractional Cable Equation, Applied Mathematical Modelling, 36(9), 4027-4043, (2012).

[36] Quintana-Murillo, J. and S. Yuste, An Explicit Numerical Method for the Fractional Cable Equation, International Journal of Differential Equations, 2011, (2011)

[37] Acan, O. and D. Baleanu, A New Numerical Technique for Solving Fractional Partial Differential Equations, arXiv preprint arXiv: 1704.02575, (2017).

[38] Acan, O., et al., Conformable Variational Iteration Method, New Trends in Mathematical Sciences, 5(5), 172-178, (2017). 